\title{
Percutaneous coronary intervention in the elderly with ST-segment elevation myocardial infarction
}

\author{
This article was published in the following Dove Press journal: \\ Clinical Interventions in Aging \\ 31 July 2014 \\ Number of times this article has been viewed
}

Lei Gao*

Xin $\mathrm{Hu}^{*}$

Yu-Qi Liu

Qiao Xue

Quan-Zhou Feng

Institute of Geriatric Cardiology,

Chinese PLA General Hospital,

Beijing, People's Republic of China

*These authors contributed

equally to this work
Correspondence: Quan-Zhou Feng Department of Cardiology, Chinese PLA General Hospital, 28 Fuxing Road, Haidian District, Beijing 100853,

People's Republic of China

Tel +86 I0 55499339

$\mathrm{Fax}+861055499339$

Email fqz30I@yahoo.com
Abstract: As a result of increased life expectancy, octogenarians constitute an increasing proportion of patients admitted to hospital for ST-segment elevation myocardial infarction (STEMI). Primary percutaneous coronary intervention is currently the treatment of choice for octogenarians presenting with STEMI. The recent literature on this topic has yielded controversial results, even though advances in drug-eluting stents and new types of antithrombotic agents are improving the management of STEMI and postoperative care. In this paper, we review the current status of percutaneous coronary intervention in the elderly with STEMI, including the reasons for their high mortality and morbidity, predictors of mortality, and strategies to improve outcomes. Keywords: octogenarians, percutaneous coronary intervention, ST-segment elevation myocardial infarction

\section{Introduction}

Primary percutaneous coronary intervention (PCI) is currently the treatment of choice for patients presenting with ST-segment elevation myocardial infarction (STEMI). With improvements in health care, the life expectancy of the elderly population has increased. The proportion of octogenarians in the general population is expected to triple by the year 2050. ${ }^{1}$ Even though octogenarians constitute an important high-risk subgroup of patients with STEMI, they are frequently under-represented in clinical trials, and advanced age is considered an independent risk factor for the early morbidity and mortality associated with PCI for STEMI. ${ }^{2,3}$ Poorer outcome is influenced not only by extensive coronary artery disease but also by more complex comorbidities. ${ }^{4}$ In addition, elderly patients are considered more likely than younger patients to suffer complications following revascularization procedures. ${ }^{5}$ The issue of PCI in octogenarians with STEMI is attracting increasing attention. In this review, we identified relevant papers from Medline and PubMed using the search terms "octogenarians", "percutaneous coronary intervention", and "ST-segment elevation myocardial infarction". The references of these papers were also searched for relevant studies in the elderly. We included English language reports published up to March 2014 that provided data for PCI in octogenarians with STEMI. The review included the reasons for the high mortality and morbidity associated with PCI in octogenarians with STEMI, predictors of mortality, and strategies to improve outcomes.

\section{Current status of primary $\mathrm{PCl}$ in the elderly with STEMI}

Many retrospective studies comparing primary PCI with medical therapy in octogenarians with STEMI show that successful primary PCI in STEMI patients older than 75 years resulted in a reduction of early and long-term mortality when compared with patients treated medically. ${ }^{6-8}$ Recently, the Western Denmark Heart Registry analysis of primary 
PCI in octogenarians and nonagenarians with STEMI showed that a total of 1,322 elderly patients were treated with primary PCI, which corresponds to $11.6 \%$ of the total STEMI population treated with primary PCI. ${ }^{9}$ In another study, performed in Turkey, Oduncu et $\mathrm{al}^{10}$ reported that $8.1 \%$ of the primary PCI population consisted of patients aged $\geq 80$ years. However, recent temporal trend studies indicate that primary PCI does not significantly improve short-term and one-year survival rates in this high-risk population. Claessen et al reviewed 379 octogenarians ( $8.4 \%$ of the total population with STEMI) treated with primary PCI between 1997 and 2007. In the total cohort of patients ( $n=379), 30$-day mortality was $21 \%$ $(n=81)$ and one-year mortality was $28 \%(n=107)$. There was no improvement in survival among octogenarian STEMI patients over the 11-year study period. ${ }^{11}$ Yamanaka et al retrospectively analyzed 1,494 octogenarians with acute myocardial infarction who underwent PCI with drug-eluting stents. They found that rates of one-year all-cause death were significantly higher in octogenarians than in nonoctogenarians (22.3\% versus $6.5 \% ; P<0.001) .{ }^{12}$ Therefore, there remains significant controversy with regard to treating octogenarians with STEMI, given the observed risk of complications with increasing age and the paucity of trials demonstrating the benefits of PCI in the octogenarian cohort. Further randomized clinical trials aiming to identify the optimal STEMI management strategy for octogenarians are warranted.

\section{Is primary $\mathrm{PCl}$ better than thrombolysis in the elderly?}

To date, only three randomized studies have specifically addressed the issue of primary PCI versus fibrinolysis in the elderly. In the Zwolle ${ }^{13}$ study, the 46 patients allocated to primary PCI showed a lower 2-year mortality rate when compared with those treated by streptokinase (15\% versus $32 \%$, respectively; $P=0.04$ ). The larger as yet unpublished Senior PAMI (Primary Angioplasty in Myocardial Infarction) trial (ClinicalTrials.gov identifier NCT00136929), ${ }^{14,15}$ which included 481 patients older than 70 years, failed to document a difference between primary PCI and fibrinolysis with regard to primary outcome (30-day mortality or stroke) or mortality. TRIANA (TRatamiento del Infarto Agudo de miocardio eN Ancianos [Treatment of acute myocardial infarction in the elderly]) was a randomized multicenter, open-label, clinical trial that included patients aged $\geq 75$ years who presented with STEMI within the first 6 hours after symptom onset. ${ }^{16}$ The primary endpoint was reached in $25(18.9 \%)$ patients in the primary PCI group and $34(25.4 \%)$ in the fibrinolysis group (odds ratio [OR] 0.69; 95\% confidence interval [CI] 0.38-1.23;
$P=0.21)$. Similarly, nonsignificant reductions were found in rates of death $(13.6 \%$ versus $17.2 \% ; P=0.43)$, reinfarction (5.3\% versus $8.2 \% ; P=0.35)$, and disabling stroke $(0.8 \%$ versus $3.0 \% ; P=0.18)$. Recurrent ischemia was less common in patients treated by primary PCI $(0.8 \%$ versus $9.7 \% ; P<0.001)$. A pooled analysis of the above three randomized controlled trials performed in very old patients showed an advantage of primary PCI over fibrinolysis in reducing death, reinfarction, and stroke at 30 days (OR 0.64; 95\% CI 0.45-0.91). ${ }^{16}$

In a study including 130 consecutive elderly patients, Goldenberg et $\mathrm{al}^{17}$ found that primary angioplasty with routine stenting in elderly patients with acute myocardial infarction was associated with better clinical outcomes and a lower risk of bleeding complications when compared with thrombolysis. The community-based perspective study by Mehta et al in the elderly (aged $\geq 70$ years) with STEMI showed that primary PCI was associated with a decrease in reinfarction and mortality when compared with thrombolytic therapy. ${ }^{18}$ In a cohort study that included 37,983 patients (aged 65 years or older) with acute myocardial infarction, ${ }^{19}$ after adjustment for demographic, clinical, hospital, and physician factors, as well as cointerventions, only primary angioplasty was associated with better survival at 30 days, although both thrombolytic therapy and primary angioplasty were associated with a survival benefit at one year when compared with no therapy.

Taking all these data together, we conclude that primary PCI may offer a clinical advantage over fibrinolytic therapy in octogenarians. However, we need to address two points regarding reperfusion therapy in octogenarians with STEMI. First, when a skilled PCI operator/team is available and can perform the invasive procedure without delay (ie, door to balloon time $<90$ minutes or within one hour of administration of fibrinolysis), it is preferable to take the STEMI patients to the catheterization laboratory rather than administer fibrinolysis. ${ }^{20}$ Second, when the diagnosis is in doubt, primary PCI is clearly preferred; not only does it provide important diagnostic information regarding the patient's symptoms, but it also decreases the risk of major bleeding associated with fibrinolysis.

\section{Reasons for high mortality and morbidity in the elderly with STEMI}

The high mortality rates in this patient population can be explained as follows. First, the effects of advancing age itself, including decreased vascular compliance, ventricular hypertrophy and remodeling, diastolic dysfunction, and diminished response to adrenergic stimulation, increase the risk of heart failure and cardiogenic shock in octogenarians 
with STEMI. ${ }^{21}$ Second, their presenting symptoms may be atypical. Electrocardiographic changes are more often equivocal in the elderly, because of, eg, pre-existing bundle branch block or residual ST-elevation from previous infarcts wall, and make electrocardiographic interpretation challenging. ${ }^{22}$ Cardiac troponin elevation can be seen in conditions other than STEMI, and many of these conditions are increasingly prevalent with age. ${ }^{23}$ All of these factors can increase the time between onset of symptoms and primary PCI therapy, thus adversely affecting the outcome. ${ }^{5}$ Third, during the intervention procedure, octogenarians are more likely to have a suboptimal angiographic result (post-procedural TIMI [Thrombolysis In Myocardial Infarction] flow grade <3) after primary PCI. Claessen et $\mathrm{al}^{11}$ found significantly lower postprocedural TIMI flow grade 3 rates in octogenarians receiving primary PCI compared with younger patients $(78 \%$ versus $92 \%$, respectively; $P<0.01$ ). Fourth, it is not surprising that octogenarians are more likely to have coexisting acute and chronic medical problems. ${ }^{24}$ An increased prevalence of significant peripheral vascular disease augments the risk of vascular complications during PCI. ${ }^{25}$ The increased prevalence of chronic kidney disease in octogenarians increases the risk of acute renal dysfunction after primary PCI. ${ }^{26}$ Further, the larger number of elderly with diabetes and multivessel coronary heart disease contributes to both procedural complexity and the overall prognosis. Metabolic abnormalities may not be recognized until primary PCI is performed, even though such comorbid conditions may significantly affect the overall management and outcome. ${ }^{27,28}$ Finally, shock is the strongest predictor of unadjusted one-year mortality, with an OR of $9.37 .^{5}$ A higher percentage of octogenarians were reported to present with shock (12\%) compared with those aged younger than 80 years $(8 \%){ }^{20}$

\section{Predictors of mortality in the elderly with STEMI}

Octogenarians have significant high-risk baseline demographic and clinical features, such as diabetes, hypertension, renal failure, anemia, cardiogenic shock, cognitive dysfunction, peripheral artery disease, longer door to balloon time, higher baseline brain natriuretic peptide, and higher C-reactive protein levels. ${ }^{12,24,29}$ Further, elderly patients not only have preinterventional characteristics that are high risk for major cardiac events, but also have poor interventional characteristics, including tortuous peripheral arteries, more severe diffuse and calcified coronary artery disease, worse interventional success rates, and lower rates of post-procedural TIMI 3 blood flow and ST-segment resolution. ${ }^{11,29}$ However, among so many risk factors, which are the most important independent predictors of mortality? Recent studies have evaluated the predictors of clinical outcome after primary PCI in octogenarians with STEMI. A Dutch study performed in an unselected, consecutive, complete cohort of octogenarians receiving primary PCI and a matched control group revealed that the most direct predictor of 30-day mortality as well as one-year mortality was cardiogenic shock at initial admission (present in 18 octogenarians versus eleven nonoctogenarians). Moreover, age and post-procedural TIMI flow $<3$ were independent predictors of mortality at 30 days and one year. ${ }^{30}$ Sakai et $\mathrm{al}^{31}$ found that overt cardiogenic shock on arrival, anterior myocardial infarction, unsuccessful reperfusion, age $>85$ years, and female sex were independent predictors of 30-day mortality in a Japanese study of primary PCI for STEMI in the elderly aged $\geq 75$ years. Tok et $\mathrm{al}^{32}$ found that independent predictors of major adverse cardiovascular events after primary PCI in octogenarians included Killip class at admission (OR 4.98, 95\% CI 1.25-19.8; $P=0.02$ ) and TIMI flow rate after primary PCI (OR 3.42, 95\% CI 1.19-10.76; $P=0.04$ ). In a report from the American College of Cardiology National Cardiovascular Data Registry, the variables associated with increased mortality were increasing age, recent myocardial infarction and its timing relative to the $\mathrm{PCI}$ procedure, renal failure, lung disease, peripheral vascular disease, and ejection fraction $<60 \%{ }^{33}$

Overall, hemodynamic disturbances, including heart failure, cardiogenic shock, and TIMI flow grade after primary PCI are the most important independent predictors of mortality in octogenarians receiving primary PCI.

\section{Strategies to improve outcomes}

With continued refinements in preprocedural evaluation and preparation, the revolution in primary PCI techniques, prevention of severe complications after PCI, and improved post-procedural management, primary PCI can be performed in octogenarians with acceptable results. Pagé et $\mathrm{al}^{34}$ described temporal trends in revascularization and outcomes after acute myocardial infarction among the very elderly. Their study found that, between March 1996 and March 2007, use of PCI increased from $2.2 \%$ to $24.9 \%$, and evidence-based prescriptions of medication increased over time $(P<0.001)$. One-year mortality improved over time $(46.5 \%$ for $1996-1999$ versus $40.9 \%$ for 2003-2006; $P<0.001$ ), while the prevalence of reported comorbidities was higher during the 2003-2006 period than during the 1996-1999 period. Although some pre-existing risk factors could not be modified or corrected in a timely manner, several strategies could still be used to improve the outcome. 


\section{Preintervention evaluation}

Physicians must be aware that primary PCI is an effective revascularization strategy to treat octogenarians with STEMI, but careful preintervention evaluation, early detection of anemia, infection, peripheral vascular disease, and kidney dysfunction, measures to decrease the volume of contrast medium used during the intervention, and particularly prevention of access site bleeding should be regarded as being as important as opening the coronary artery, particularly considering the effects of these factors on long-term mortality.

\section{Prevention of major bleeding}

Patients over 80 years of age with STEMI undergoing primary PCI are at high risk for bleeding complications. ${ }^{35}$ Oduncu et $\mathrm{al}^{10}$ reported that half of their very elderly patients with STEMI had baseline anemia, with $9.5 \%$ having major bleeding and $19 \%$ needing transfusion during the in-hospital period. The CathPCI Registry study ${ }^{36}$ evaluated trends in use of bleeding avoidance strategies (direct thrombin inhibitors, vascular closure devices, and radial access) and risk-adjusted bleeding over time. Of 10,469 patients aged older than 80 years undergoing primary PCI, 1,002 (9.6\%) developed a bleeding complication. In multivariable analysis, use of bleeding avoidance strategies was associated with a lower risk of bleeding.

Recent studies showed that transradial primary percutaneous intervention, particularly by preventing access site bleeding, was associated with a significant mortality advantage when compared with the femoral approach. ${ }^{37}$ The RIVAL (Radial versus femoral access for coronary angiography and intervention in patients with acute coronary syndromes) (ClinicalTrials.gov identifier NCT01014273) investigators reported a significantly lower rate of vascular complications in their randomized, parallel-group, multicenter study of patients undergoing PCI for STEMI, resulting in a reduction in overall mortality in the radial access group. ${ }^{38}$ The transradial approach may also increase the success rate of primary PCI in the elderly by circumventing the procedural problems related to peripheral artery disease (which is very frequent in those over 80 years of age), and may overcome the difficulty in passing through tortuous femoral, iliac, abdominal, and thoracic aortae.

Accordingly, antithrombotic treatment should also be tailored in patients over 80 years of age to prevent major bleeding events. Bivalirudin, a direct thrombin inhibitor, has gained a Class $\mathrm{Ib}$ recommendation as a supportive measure for primary PCI. ${ }^{39}$ A prespecified age subgroup analysis of the ACUITY (Acute Catheterization and Urgent Intervention Triage Strategy) study has recently demonstrated that although there were no significant differences in efficacy, the benefit of bivalirudin in terms of absolute reduction in bleeding events is most pronounced in patients $>75$ years of age..$^{40}$

Overall, the presently established strategies to prevent major bleeding in octogenarians include adjusting doses of glycoprotein IIb/IIIa inhibitors, using enoxaparin for patients with renal insufficiency, considering use of bivalirudin for primary PCI, considering low-dose aspirin $(81 \mathrm{mg})$ for chronic antiplatelet therapy, and avoiding triple anticoagulant therapy (aspirin, clopidogrel, warfarin) when possible. Potential strategies include adjusting doses of aspirin and clopidogrel based upon point-of-care platelet function assays and selecting the radial artery routinely for arterial access.

\section{Prevention of contrast-induced acute kidney injury}

Contrast-induced acute kidney injury is known to be associated with both urgent procedures and advanced age. The volume of contrast medium used during urgent coronary intervention tends to be relatively higher than in elective procedures. Accordingly, adequate hydration before and after exposure to contrast medium, along with avoidance of nephrotoxic drugs, remains the recommended prophylaxis for acute kidney injury. ${ }^{41}$ Recent clinical evidence does not support the use of $\mathrm{N}$-acetylcysteine for prophylaxis against acute kidney injury. ${ }^{42,43}$

\section{Treatment of cardiogenic shock}

Cardiogenic shock is a complication in approximately $5 \%-8 \%$ of patients presenting with $\mathrm{STEMI}^{44}$ and remains the leading cause of death after hospitalization. Although we emergently revascularize occluded coronaries in cardiogenic shock (SHOCK: SHould we emergently revascularize Occluded Coronaries in cardiogenic shocK) trial, ${ }^{20}$ no benefit of revascularization was found in patients over 75 years of age. However, several recent larger observational studies have shown benefits of early revascularization in the elderly with cardiogenic shock..$^{45,46}$ In the large SHOCK registry (1993-1997), ${ }^{45}$ which included 277 elderly patients, in-hospital mortality for patients aged $\geq 75$ years was $48 \%$ with early revascularization (within 18 hours of myocardial infarction) compared with $81 \%$ in the late or no revascularization group $(P=0.0002)$. Similarly, a study of cardiogenic shock at the Mayo Clinic between 1991 and 2000 evaluated patients aged $\geq 75$ years $(n=61)$ undergoing urgent PCI, and the in-hospital mortality rate was $44 \%$ with a 30 -day mortality of $47 \% .{ }^{46}$ The high rate of early reperfusion, stent deployment, and glycoprotein $\mathrm{IIb} / \mathrm{III}$ inhibitor use may be the reasons for the comparable outcomes between octogenarians and younger patients. These data suggest that benefit is possible with selective use 
of early revascularization in octogenarians presenting with cardiogenic shock, and more prospective studies need to be performed for further conclusions.

\section{Prevention of slow reflow or no reflow}

Slow reflow and no reflow are frequent findings in patients with STEMI treated by primary PCI. Octogenarians are more likely to have a suboptimal angiographic result (postprocedural TIMI flow grade $<3$ ) after primary PCI. In recent years, various pharmacologic and nonpharmacologic approaches have been used as adjunctive therapy to PCI to enhance myocardial reperfusion and salvage and to improve the outcome in patients with STEMI. Although many types of medication, including adenosine, sodium nitroprusside, nicorandil, and other agents have recently been tested as adjunctive therapy to PCI in patients with STEMI, ${ }^{47}$ the available evidence is not strong enough to recommend that any of these interventions be routinely used as an adjunct to primary PCI in patients with STEMI.

Apart from the abovementioned pharmacologic agents, mechanical devices have been developed to prevent distal embolization. Although a meta-analysis ${ }^{48}$ supports the use of catheter thrombus aspiration during primary PCI to improve microvascular reperfusion and to decrease deaths and adverse cardiac events, there is no strong evidence that distal embolization protection and manual aspiration devices can effectively prevent distal embolization, ${ }^{49,50}$ at best, no evidence in the elderly.

On the basis of the current evidence, the decision to perform primary PCI in the elderly should not be based on chronological age alone, but rather on each patient's general eligibility for revascularization and the clinical circumstances as a whole.

In summary, primary PCI is currently the most preferred reperfusion strategy in the elderly; however, it is widely accepted that primary PCI in the elderly is more challenging for a variety of reasons, such as their more complex coronary anatomy, the increased rate of bleeding complications, increased rate of vascular complications, and increased prevalence of comorbidities. Future prospective studies in the elderly, especially octogenarians, with STEMI are needed in order to evaluate the effectiveness and safety of primary $\mathrm{PCI}$ in this patient population.

\section{Disclosure}

The authors report no conflicts of interest in this work.

\section{References}

1. Centers for Disease Control and Prevention. MMWR series on public health and aging. MMWR Morb Mortal Wkly Rep. 2003;52(6):101-116.

2. Singh M, Mathew V, Garratt KN, et al. Effect of age on the outcome of angioplasty for acute myocardial infarction among patients treated at the Mayo Clinic. Am J Med. 2000;108(3):187-192.
3. Newell MC, Henry JT, Henry TD, et al. Impact of age on treatment and outcomes in ST-elevation myocardial infarction. Am Heart J. 2011; 161(4):664-672.

4. Yang $\mathrm{X}$, Zhao $\mathrm{Y}, \mathrm{Wu} \mathrm{H}$, et al. The coexistence of comorbidities at admission is an independent predictor of 30-day mortality of patients hospitalized with acute myocardial infarction: analysis of 5523 cases in China. Int J Cardiol. 2012;155(3):451-452.

5. Alexander KP, Newby LK, Armstrong PW, et al. Acute coronary care in the elderly, part II: ST-segment-elevation myocardial infarction: a scientific statement for healthcare professionals from the American Heart Association Council on Clinical Cardiology: in collaboration with the Society of Geriatric Cardiology. Circulation. 2007;115(19): 2570-2589.

6. Wang YC, Hwang JJ, Hung CS, Kao HL, Chiang FT, Tseng CD. Outcome of primary percutaneous coronary intervention in octogenarians with acute myocardial infarction. J Formos Med Assoc. 2006;105(6): 451-458.

7. Skelding KA, Mehta LS, Pica MC, et al. Primary percutaneous interventions for acute myocardial infarction in octogenarians: a single-center experience. Clin Cardiol. 2002;25(8):363-366.

8. Sosnowski C, Janeczko-Sosnowska E, Wozniak J, et al. Primary coronary intervention in diabetic octogenarians with acute ST elevation myocardial infarction. Kardiol Pol. 2007;65(10):1181-1186.

9. Antonsen L, Jensen LO, Terkelsen CJ, et al. Outcomes after primary percutaneous coronary intervention in octogenarians and nonagenarians with ST-segment elevation myocardial infarction: from the Western Denmark Heart Registry. Catheter Cardiovasc Interv. 2013;81(6):912-919.

10. Oduncu V, Erkol A, Tanalp AC, et al. [Comparison of early and late clinical outcomes in patients $>=80$ versus $<80$ years of age after successful primary angioplasty for ST segment elevation myocardial infarction]. Turk Kardiyol Dern Ars. 2013;41(4): 319-328. Turkish.

11. Claessen BE, Kikkert WJ, Engstrom AE, et al. Primary percutaneous coronary intervention for ST elevation myocardial infarction in octogenarians: trends and outcomes. Heart. 2010;96(11):843-847.

12. Yamanaka F, Jeong MH, Saito S, et al. Comparison of clinical outcomes between octogenarians and non-octogenarians with acute myocardial infarction in the drug-eluting stent era: analysis of the Korean Acute Myocardial Infarction Registry. J Cardiol. 2013;62(4): 210-216.

13. de Boer MJ, Ottervanger JP, van 't Hof AW, Hoorntje JC, Suryapranata H, Zijlstra F. Reperfusion therapy in elderly patients with acute myocardial infarction: a randomized comparison of primary angioplasty and thrombolytic therapy. J Am Coll Cardiol. 2002;39(11): 1723-1728.

14. ClinicalTrials.gov. Senior PAMI: Primary angioplasty versus thrombolytic therapy for acute myocardial infarction in the elderly. May 14, 2010. Available from: http://www.clinicaltrial.gov/ct2/show/ NCT00136929. Accessed June 19, 2014.

15. TheHeart.Org. O'Riordan M. SENIOR PAMI: primary PCI not better than lytic therapy in elderly patients. May 14, 2010. Available from: www.theheart.org/article/581549.do. Accessed June 19, 2014.

16. Bueno H, Betriu A, Heras M, et al. Primary angioplasty vs fibrinolysis in very old patients with acute myocardial infarction: TRIANA (TRatamiento del Infarto Agudo de miocardio eN Ancianos) randomized trial and pooled analysis with previous studies. Eur Heart J. 2011; 32(1):51-60.

17. Goldenberg I, Matetzky S, Halkin A, et al. Primary angioplasty with routine stenting compared with thrombolytic therapy in elderly patients with acute myocardial infarction. Am Heart J. 2003;145(5): 862-867.

18. Mehta RH, Sadiq I, Goldberg RJ, et al. Effectiveness of primary percutaneous coronary intervention compared with that of thrombolytic therapy in elderly patients with acute myocardial infarction. Am Heart J. 2004;147(2):253-259.

19. Berger AK, Radford MJ, Wang Y, Krumholz HM. Thrombolytic therapy in older patients. J Am Coll Cardiol. 2000;36(2):366-374.

20. Hochman JS, Sleeper LA, Webb JG, et al. Early revascularization in acute myocardial infarction complicated by cardiogenic shock. SHOCK Investigators. Should we emergently revascularize occluded coronaries for cardiogenic shock. N Engl J Med. 1999;341(9):625-634. 
21. Carro A, Kaski JC. Myocardial infarction in the elderly. Aging Dis. 2011; 2(2):116-137.

22. Dangas GD, Singh HS. Primary percutaneous coronary intervention in octogenarians: navigate with caution. Heart. 2010;96(11):813-814.

23. Inbar R, Shoenfeld $Y$. Elevated cardiac troponins: the ultimate marker for myocardial necrosis, but not without a differential diagnosis. Isr Med Assoc J. 2009;11(1):50-53.

24. Picarra BC, Santos AR, Celeiro M, et al. Non-cardiac comorbidities in the very elderly with acute myocardial infarction: prevalence and influence on management and in-hospital mortality. Rev Port Cardiol. 2011;30(4):379-392.

25. Renilla A, Barreiro M, Barriales V, Torres F, Alvarez P, Lambert JL. Management and risk factors for mortality in very elderly patients with acute myocardial infarction. Geriatr Gerontol Int. 2013;13(1): 146-151.

26. Zhao WS, Li KB, Zhang Y, Wang HS, Wang LF, Yang XC. [The inhospital mortality and its determinants for very elderly patients with acute myocardial infarction]. Zhonghua Nei Ke Za Zhi. 2011;50(12): 1023-1025. Chinese.

27. Timoteo AT, Ramos R, Toste A, et al. [Impact of age on treatment and outcomes after acute myocardial infarction, particularly in very elderly patients]. Rev Port Cardiol. 2011;30(12):897-903. Portuguese.

28. Okmen E. [Primary percutaneous coronary intervention in octogenarians]. Turk Kardiyol Dern Ars. 2013;41(4):329-331. Turkish.

29. DeGeare VS, Stone GW, Grines L, et al. Angiographic and clinical characteristics associated with increased in-hospital mortality in elderly patients with acute myocardial infarction undergoing percutaneous intervention (a pooled analysis of the primary angioplasty in myocardial infarction trials). Am J Cardiol. 2000;86(1):30-34.

30. Moonen LA, van 't Veer M, Pijls NH. Procedural and long-term outcome of primary percutaneous coronary intervention in octogenarians. Neth Heart J. 2010;18(3):129-134.

31. Sakai K, Nagayama S, Ihara K, et al. Primary percutaneous coronary intervention for acute myocardial infarction in the elderly aged $>/=75$ years. Catheter Cardiovasc Interv. 2012;79(1):50-56.

32. Tok D, Turak O, Ozcan F, et al. [Primary percutaneous coronary intervention for acute myocardial infarction in elderly aged 75 years and over: in-hospital mortality and clinical outcome]. Turk Kardiyol Dern Ars. 2012;40(7):565-573. Turkish.

33. Wang TY, Masoudi FA, Messenger JC, et al. Percutaneous coronary intervention and drug-eluting stent use among patients $\geq 85$ years of age in the United States. J Am Coll Cardiol. 2012;59(2):105-112.

34. Page M, Doucet M, Eisenberg MJ, Behlouli H, Pilote L. Temporal trends in revascularization and outcomes after acute myocardial infarction among the very elderly. CMAJ. 2010;182(13):1415-1420.

35. Kinnaird T, Anderson R, Hill J, Thomas M. Bleeding during percutaneous intervention: tailoring the approach to minimise risk. Heart. 2009; 95(1):15-19.

36. Dodson JA, Wang Y, Chaudhry SI, Curtis JP. Bleeding-avoidance strategies and outcomes in patients $\geq 80$ years of age with ST-elevation myocardial infarction undergoing primary percutaneous coronary intervention (from the NCDR CathPCI Registry). Am J Cardiol. 2012;110(1):1-6.
37. Arzamendi D, Ly HQ, Tanguay JF, et al. Effect on bleeding, time to revascularization, and one-year clinical outcomes of the radial approach during primary percutaneous coronary intervention in patients with STsegment elevation myocardial infarction. Am J Cardiol. 2010;106(2): $148-154$.

38. Jolly SS, Yusuf S, Cairns J, et al. Radial versus femoral access for coronary angiography and intervention in patients with acute coronary syndromes (RIVAL): a randomised, parallel group, multicentre trial. Lancet. 2011;377(9775):1409-1420.

39. O'Gara PT, Kushner FG, Ascheim DD, et al. 2013 ACCF/AHA guideline for the management of ST-elevation myocardial infarction: a report of the American College of Cardiology Foundation/American Heart Association Task Force on Practice Guidelines. Circulation. 2013; 127(4):e362-e425.

40. Lopes RD, Alexander KP, Manoukian SV, et al. Advanced age, antithrombotic strategy, and bleeding in non-ST-segment elevation acute coronary syndromes: results from the ACUITY (Acute Catheterization and Urgent Intervention Triage Strategy) trial. J Am Coll Cardiol. 2009; 53(12):1021-1030.

41. Sadat U. N-acetylcysteine in contrast-induced acute kidney injury: clinical use against principles of evidence-based clinical medicine. Expert Rev Cardiovasc Ther. 2014;12(1):1-3.

42. ACT Investigators. Acetylcysteine for prevention of renal outcomes in patients undergoing coronary and peripheral vascular angiography: main results from the randomized Acetylcysteine for Contrast-induced nephropathy Trial (ACT). Circulation. 2011;124(11):1250-1259.

43. Erturk M, Uslu N, Gorgulu S, et al. Does intravenous or oral high-dose $\mathrm{N}$-acetylcysteine in addition to saline prevent contrast-induced nephropathy assessed by cystatin C. Coron Artery Dis. 2014;25(2):111-117.

44. Reynolds HR, Hochman JS. Cardiogenic shock: current concepts and improving outcomes. Circulation. 2008;117(5):686-697.

45. Dzavik V, Sleeper LA, Cocke TP, et al. Early revascularization is associated with improved survival in elderly patients with acute myocardial infarction complicated by cardiogenic shock: a report from the SHOCK Trial Registry. Eur Heart J. 2003;24(9):828-837.

46. Migliorini A, Moschi G, Valenti R, et al. Routine percutaneous coronary intervention in elderly patients with cardiogenic shock complicating acute myocardial infarction. Am Heart J. 2006;152(5):903-908.

47. Iwakura $\mathrm{K}$, Ito $\mathrm{H}$, Okamura $\mathrm{A}$, et al. Nicorandil treatment in patients with acute myocardial infarction: a meta-analysis. Circ J. 2009;73(5): 925-931.

48. Bavry AA, Kumbhani DJ, Bhatt DL. Role of adjunctive thrombectomy and embolic protection devices in acute myocardial infarction: a comprehensive meta-analysis of randomized trials. Eur Heart J. 2008; 29(24):2989-3001.

49. Parodi G, Valenti R, Migliorini A, et al. Comparison of manual thrombus aspiration with rheolytic thrombectomy in acute myocardial infarction. Circ Cardiovasc Interv. 2013;6(3):224-230.

50. Jolly SS, Bertrand OF. Another chapter in the story of thrombectomy in ST-elevation myocardial infarction: a story not yet finished. Eur Heart $J$. 2013;34(14):1024-1026.
Clinical Interventions in Aging

\section{Publish your work in this journal}

Clinical Interventions in Aging is an international, peer-reviewed journal focusing on evidence-based reports on the value or lack thereof of treatments intended to prevent or delay the onset of maladaptive correlates of aging in human beings. This journal is indexed on PubMed Central, MedLine,

\section{Dovepress}

CAS, Scopus and the Elsevier Bibliographic databases. The manuscript management system is completely online and includes a very quick and fair peer-review system, which is all easy to use. Visit http://www.dovepress. com/testimonials.php to read real quotes from published authors. 